\title{
Melanocortin systems on pigment dispersion in fish chromatophores
}

\author{
Yuki Kobayashi ${ }^{1,2 t}$, Kanta Mizusawa ${ }^{1}, Y_{\text {Iumiko Saito }}{ }^{2}$ and Akiyoshi Takahashi ${ }^{1 *}$ \\ ${ }^{1}$ School of Marine Biosciences, Kitasato University, Sagamihara, Kanagawa, Japan \\ ${ }^{2}$ Graduate School of Integrated Arts and Sciences, Hiroshima University, Higashi-Hiroshima, Hiroshima, Japan
}

Edited by:

Yong Zhu, East Carolina University, USA

\section{Reviewed by:}

Paula Gabriela Gabriela Vissio, Universidad de Buenos Aires, Argentina

Masazumi Sugimoto, Toho University, Japan

Jose Miguel Cerdá-Reverter, Consejo Superior de Investigaciones

Cientificas, Spain

*Correspondence:

Akiyoshi Takahashi, School of Marine Biosciences, Kitasato University,

1-15-1 Kitasato, Minami-ku,

Sagamihara, Kanagawa 252-0373,

Japan.

e-mail: akiyoshi@kitasato-u.ac.jp

${ }^{+}$Present address:

Yuki Kobayashi, Graduate School of Integrated Arts and Sciences,

Hiroshima University,

Higashi-Hiroshima, Hiroshima

739-8521, Japan.
$\alpha$-Melanocyte-stimulating hormone $(\alpha-\mathrm{MSH})$ is responsible for pigment dispersion in the chromatophores of fish and other tetrapods such as amphibians and reptiles. Recently, we discovered that $\alpha-\mathrm{MSH}$ did not always stimulate pigment dispersion because this hormonal peptide exerted no effects on the melanophores of flounders. We assumed that the reduction of $\alpha-\mathrm{MSH}$ activity was related to the co-expression of different $\alpha-\mathrm{MSH}$ receptor subtypes - termed melanocortin receptors (MCR) - a member of G-protein-coupled receptors (GPCR) - based on several reports demonstrating that GPCR forms heterodimers with various properties that are distinct from those of the corresponding monomers. In this review, we summarize the relationships between the pigment-dispersing activity of $\alpha$-MSH-related peptides, molecular forms of $\alpha-\mathrm{MSH}$-related peptides, and $\mathrm{mcr}$ subtypes expressed in fish chromatophores.

\section{Keywords: melanocyte-stimulating hormone, melanocortin, melanocortin receptor, G-protein-coupled receptor,} heterodimer, pigment-dispersing activity

\section{INTRODUCTION}

Fish color change is achieved by two major aspects, such as physiological and morphological color change. Short-term physiological color change in teleosts is caused by pigment aggregation/dispersion in skin chromatophores in which the neuroendocrine and sympathetic nervous systems are involved (Fujii and Oshima, 1986; Fujii, 2000). In the long term, these systems also influence survival or apoptosis of the chromatophores and contribute to morphological color change (Sugimoto, 2002).

The hypothalamo-pituitary system is a major player of the neuroendocrine system, in which melanocyte-stimulating hormone (MSH) and melanin-concentrating hormone $(\mathrm{MCH})$ are associated with color changes in fish, exerting opposing actions (Takahashi and Kawauchi, 2006a; Mizusawa et al., 2011). MSH stimulates pigment dispersion and $\mathrm{MCH}$ induces pigment aggregation.

Melanocyte-stimulating hormone is derived from a precursor termed proopiomelanocortin (POMC), and $\mathrm{MCH}$ is derived from a precursor called proMCH (Takahashi and Kawauchi, 2006a). The major sources of MSH and $\mathrm{MCH}$ are the pituitary gland and hypothalamus, respectively. The MSH receptor, known as the melanocortin (MC) receptor (MCR), is a member of the Gprotein-coupled receptor (GPCR) family, as is the $\mathrm{MCH}$ receptor. The members of this family have seven transmembrane domains
(Mountjoy et al., 1992; Saito et al., 1999). Five subtypes and two subtypes are present for MCR and MCH receptor, respectively (Takahashi and Kawauchi, 2006b).

Compared to the rather conservative amino acid sequence of $\mathrm{MCH}, \mathrm{MSH}$ varies in molecular form because of the presence of two or more molecular forms encoded in pomc and a variety of modifications to the N- and C-terminals (Ebelre, 1988). Recently, we discovered that the biological activities of $\alpha$-MSH-related peptides were dramatically reduced by the presence of an acetyl group, and this modulation was related to the co-expression of different $m c r$ types (Kobayashi et al., 2009, 2010). Abundant evidence regarding this interesting activity modulation was obtained in an experiment using barfin (bf) flounder, a flatfish that is commercially important in the northern part of Japan. Comparative studies using the closely related Japanese (Jpn) flounder (Kobayashi et al., 2012) and the distantly related goldfish (Kobayashi et al., 2011) further supported these results. In this review, we provide an overview of our recent studies on the relationships between $\mathrm{mcr}$ transcripts expressed in fish chromatophores and the biological activities of $\alpha$-MSH-related peptides possessing different numbers of acetyl groups. The differences in activity of these peptides appear to be related to the interaction of ligands with different degrees of acetylation at the N-terminus and the types of MCR biosynthesized in the chromatophores. 


\section{GENERAL ASPECTS OF MC SYSTEMS AND CHALLENGES PROVIDED BY $\alpha$-MSH}

Melanocortins are members of peptides such as $\alpha-\mathrm{MSH}, \beta-\mathrm{MSH}$, $\gamma-\mathrm{MSH}$, and adrenocorticotropic hormone (ACTH). These peptides are derived from a common precursor protein termed proopiomelanocortin (Smith and Funder, 1988; Castro and Morrison, 1997; Raffin-Sanson et al., 2003; Kasper et al., 2006; Takahashi and Kawauchi, 2006b). POMC also contains $\beta$-endorphin ( $\beta$-END), a non-MC peptide. In general, $\alpha-\mathrm{MSH}$ is composed of 13 amino acid residues with an acetylated $\mathrm{N}$-terminal and an amidated $\mathrm{C}$ terminal. $\alpha-\mathrm{MSH}$ is derived from the $\mathrm{N}$-terminal portion of ACTH comprising 39 amino acid residues, and other MSHs originate from different parts of POMC. While the POMCs of basal rayfinned fish, lobe-finned fish, and tetrapods contain three MSHs, those derived from ray-finned fish, namely teleosts, lack $\gamma$-MSH, but contain $\alpha$-MSH and $\beta$-MSH (Takahashi and Kawauchi, 2006a). Sharks and rays are unique because of the presence of four MSHs; the fourth MSH is termed $\delta$-MSH (Amemiya et al., 1999, 2000; Takahashi et al., 2004, 2008). $\gamma$-MSH appears to have been deleted from POMC and $\delta-\mathrm{MSH}$ is suggested to be derived from $\beta-\mathrm{MSH}$ during the evolution of teleost and cartilaginous fish, respectively (Takahashi and Kawauchi, 2006a).

A major source of POMC is the pars intermedia (neurointermediate lobe, NIL, in teleosts) and pars distalis (PD) of the pituitary gland, and the final products in each lobe are different because of tissue-specific post-translational processing (Smith and Funder, 1988; Castro and Morrison, 1997; Raffin-Sanson et al., 2003; Kasper et al., 2006; Takahashi and Kawauchi, 2006b). For example, ACTH is a product of the PD, and $\alpha-M S H$ is a product of the NIL.

The presence of five subtypes - MC1R to MC5R - has been reported for MCR (Mountjoy et al., 1992; Mountjoy, 2000; Gantz and Fong, 2003). These subtypes are distributed in bodies in a rather subtype-specific manner, and are associated with a variety of biological processes conveyed by MC peptides. Of the subtypes, MC1R and MC2R are the classical $\alpha-\mathrm{MSH}$ and ACTH receptors, respectively (Mountjoy, 2000; Gantz and Fong, 2003). Many experiments on the binding affinity of each subtype vs. a variety of MC peptides have been conducted using a solely expressed MCR molecule in a transfected cell line (Cone, 2000; Schiöth et al., 2005). These procedures have characterized a remarkable difference between MC2R and the other MCRs - while MC2R binds ACTH, but not MSH, the other MCRs bind both ACTH and MSH. These methods have also contributed to the development of many MC peptide-related antagonists and agonists (Cone, 2003). However, increasing biochemical and biophysical evidences have indicated that GPCRs have been shown to form heterodimers or heterooligomers with various biochemical and/or pharmacological activities that are distinct from those of the corresponding monomers or homomers (Satake and Sakai, 2008; Rozenfeld and Devi, 2011).

The presence or absence of an acetyl group at the N-terminal of $\alpha-\mathrm{MSH}$ is involved in the regulation of this peptide's biological activities. In frogs, pigment-dispersing activities in chromatophores are enhanced by the presence of an acetyl group (Ebelre, 1988), while desacetyl (Des-Ac)- $\alpha$-MSH stimulates lipid mobilization in the liver of rainbow trout (Yada et al., 2000). Moreover, diacetyl (Di-Ac)- $\alpha$-MSH, which has acetyl groups at the $N$ and $O$ positions of the $\mathrm{N}$-terminal Ser residue, demonstrates substantial cortisol-releasing activity on the head kidney in tilapia (Lamers et al., 1992). This activity is followed by Des-Ac- $\alpha-\mathrm{MSH}$ and $\alpha-\mathrm{MSH}$ in order of potency. Namely, these results suggest that the number of acetyl groups on $\alpha$-MSH-related peptides may influence ligand-receptor interactions, and that the biological activities of these peptides are not always proportional to the degree of acetylation. These findings are also different from pharmacological properties demonstrating that the interactions of $\alpha-\mathrm{MSH}$ and $\mathrm{Di}-\mathrm{Ac}-\alpha-\mathrm{MSH}$ via solely expressed MCRs are greater than those of Des-Ac- $\alpha-$ MSH (Schiöth et al., 1996; Sánchez et al., 2009a,b, 2010). We have obtained evidence solving these paradoxical results using bf flounder as well as other fish such as Jpn flounder and goldfish, as described farther on.

\section{POMC AND MC PEPTIDES IN FLOUNDERS}

While most teleosts have two POMC subtypes, probably caused by gene duplication events during fish evolution, we first reported that three pomc subtypes are expressed in the pituitary gland of bf flounders (Takahashi et al., 2005). Two of the POMC types (A and $\mathrm{B})$ contain two MC sequences corresponding to $\alpha$ - and $\beta$ $\mathrm{MSH}$, and $1 \beta$-END, as is the case for other teleosts. The third POMC, namely POMC-C, is composed of $\alpha$ - and $\beta-\mathrm{MSH}$, and a $\beta$-END remnant, which appears to be secondarily mutated after divergence from the strain leading to POMC-A and - $\mathrm{B}$. Therefore, it is conceivable that POMC-C may have lost the bifunctional role as a precursor for both $\mathrm{MC}$ peptides and $\beta$-END, and became specialized for the precursor of MC peptides, the so-called promelanocortin, as we previously mentioned (Takahashi et al., 2005; Takahashi and Kawauchi, 2006a). While the amino acid sequence of $\alpha-\mathrm{MSH}$ - including $\alpha-\mathrm{MSH}-\mathrm{A}$ and -B derived from bf flounder POMC-A and -B, respectively - is most conserved among the several MSH types, bf flounder $\alpha-\mathrm{MSH}-\mathrm{C}$ derived from POMC-C differs by one residue with respect to the sequences of $\alpha-\mathrm{MSH}-$ at position 13 (Thr-amide in $\alpha-\mathrm{MSH}-\mathrm{C}$ vs. Val-amide $\alpha-\mathrm{MSH}$; Takahashi et al., 2005).

Pomcs are expressed in both the PD and NIL of the bf flounder pituitary gland (Takahashi et al., 2006). In these lobes, POMCs are cleaved into several biologically active peptides. The major products in the PD are ACTH, while a part of ACTH is further cleaved into Des-Ac- $\alpha-\mathrm{MSH}$, and those in the NIL are Des-Ac- $\alpha-\mathrm{MSH}$, $\alpha-\mathrm{MSH}$, Di-Ac- $\alpha$-MSH, $\beta$-MSH, and C-terminally truncated $N$ Ac- $\beta$-END. Thus, the differences between the PD and NIL reside in the final products, including the occurrence of acetylation.

The pomc- $a$ transcripts are most predominant in the pituitary, where pomc- $b$ transcripts are second in predominance (Kobayashi et al., 2009). While pomc-c is expressed in the pituitary, this gene is also expressed in the eyed side and non-eyed side skin of the flounder. While pomc-c transcripts are detected at extremely low concentrations in the skin compared to that in the pituitary, their total amounts in whole skins are comparable to those of pomc-a in whole pituitary glands. Des-Ac- $\alpha-\mathrm{MSH}-\mathrm{C}$ derived from POMC-C is also observed from bf flounder skin extracts, indicating that the skin is another major source of $\alpha$-MSH-related peptides.

Pomc-a, pomc-b, and pomc-c cDNA have been cloned in Jpn flounder (Kim et al., 2009 and accession number ACG68732); the original terms POMC-II, POMC-I, and POMC-III in the paper 
correspond to POMC-A, POMC-B, and POMC-C, respectively. We observed the expression of these three genes in the pituitary and those of pomc-c in Jpn flounder skin. Therefore, it is indicated that the pomc expression patterns of these two flounder species are closely related.

\section{Mcr SUBTYPES IN FLOUNDERS AND GOLDFISH}

The presence of $\mathrm{mcr}$ has been reported in wide vertebrate classes such as Cephalaspidomorphi (lampreys), Chondrichthyes (sharks), Sarcopterygii (lobe-finned fish, including tetrapods), and Actinopterygii (ray-finned fish; Klovins et al., 2004; Schiöth et al., 2005; Haitina et al., 2007a,b). While mammals and chickens have shown to possess five $m c r$ subtypes - $m c 1 r$ to $m c 5 r$, zebrafish is the only fish species in which the presence of a set of five subtypes was demonstrated, while its $m c 5 r$ is subdivided into $m c 5 r a$ and mc5rb (Logan et al., 2003; Klovins et al., 2004). Fugu may lack $m c 3 r$, while four $m c r$ subtypes have been identified by genomic studies. We have cloned the cDNAs for $m c 1 r, m c 2 r, m c 4 r$, and $m c 5 r$ in bf flounder (Kobayashi et al., 2008, 2010, 2011). These four $m c r$ cDNAs have also been identified in Jpn flounder (Kobayashi et al., 2012). Taking the taxonomically close relationship between Pleuronectiformes, including flounders, and Tetraodontiformes, including Fugu, into consideration, flounders may lack $m c 3 r$, as is the case with Fugu.

On the other hand, we recently demonstrated the presence of $m c 1 r, m c 2 r$, and $m c 3 r$ in goldfish (Kobayashi et al., 2011). These results, as well as the previous characterization of $m c 4 r$ and $m c 5 r$ cDNA (Cerdá-Reverter et al., 2003a,b), provide the second line of evidence for the presence of five mor subtypes in fish. The Cypriniformes, to which both goldfish and zebrafish belong, is a group of ray-finned fish that is rather basal when compared to the Fugu of Tetraodontiformes (Nelson, 2006). Therefore, it is possible that the five $m o r$ subtypes may have appeared in an early vertebrate, possibly in a common ancestor of ray-finned fish and tetrapods. $M c 3 r$ may have been deleted during the course of evolution from a strain from which ray-finned fishes such as flounder and Fugu were descended.

\section{$\alpha$-MSH ACTIVITY: RELATIONSHIP WITH ACETYLATION AND mors EXPRESSED IN CHROMATOPHORES \\ NEW CONCEPTS OBTAINED FROM bf FLOUNDER}

In teleost fish, MSHs stimulate not only melanophores but also other bright-colored chromatophores - erythrophores, xanthophores, and leucophores (Fujii and Oshima, 1986; Fujii, 2000). In classical concepts, acetylation increases the melanin-dispersing activity of $\alpha-\mathrm{MSH}$ in teleosts (Kawauchi et al., 1984) as well as amphibians (Ebelre, 1988). Moreover, $\alpha$-MSH-related peptides stimulate both melanophores and xanthophores in bf flounder; however, $\mathrm{N}$-terminal acetylation differentially modulates the pigment-dispersing activities of these cells (Kobayashi et al., 2009, 2010). Surprisingly, acetylation reduces the activity of $\alpha-\mathrm{MSH}$ on melanophores, while enhancing its activity on xanthophores (Table 1). Reduction of the pigment-dispersing activity of $\alpha-\mathrm{MSH}$ by acetylation is the first piece of evidence for this. On the other hand, replacement of the C-terminal residue of $\alpha$-MSHs may have negligible effects on pigment-dispersing activities because DesAc- $\alpha-\mathrm{MSH}-\mathrm{C}$ and Des-Ac- $\alpha-\mathrm{MSH}$ exhibit similar effects on the two chromatophore types (Kobayashi et al., 2009). We expect the differences in the effects of $\alpha$-MSHs on pigment dispersion with modification of the $\mathrm{N}$-terminal residue to provide insight into the interaction of $\alpha-\mathrm{MSH}$-related peptides with MCRs.

In the early stage of our investigation into MC systems in fish skin, preliminary findings detected the expression of $m c 1 r, m c 4 r$, and $m c 5 r$ in bf flounder skin parts (Kobayashi et al., 2007). Based on these results, we assumed that different pigment-dispersing activities between Des-Ac- $\alpha-\mathrm{MSH}$ and $\alpha$-MSH were results from the difference in MCR subtypes generated between xanthophores and melanophores. In brief, MCR(s) on xanthophores might interact with $\alpha$-MSHs irrespective of the presence or absence of an acetyl group, demonstrating higher sensitivities to $\alpha$-MSH than to Des-Ac- $\alpha-\mathrm{MSH}$. With the exception of MC2R, a specific receptor of $\mathrm{ACTH}, \alpha-\mathrm{MSH}$ has demonstrated the highest affinity to MC1R among the MCRs in pharmacological studies using human MCRs (Schiöth et al., 1997). Moreover, Des-Ac- $\alpha-M S H$ demonstrated a higher affinity to MC4R and MC5R than to MC1R

Table 1 | Mcr subtypes expressed in fish chromatophores and pigment-dispersing activities of $\alpha$-MSH-related peptides in these cells.

\begin{tabular}{|c|c|c|c|c|}
\hline \multirow[t]{2}{*}{ Chromatophores } & \multirow[t]{2}{*}{ Mcr subtypes } & \multicolumn{3}{|c|}{ Pigment-dispersing activities } \\
\hline & & Des & Mono & Di \\
\hline \multicolumn{5}{|c|}{ BARFIN FLOUNDER ${ }^{1}$} \\
\hline Melanophores & 1 and 5 & + & - & $\mathrm{N}$ \\
\hline Xanthophores & 5 & + & ++ & $\mathrm{N}$ \\
\hline \multicolumn{5}{|c|}{ JAPANESE FLOUNDER ${ }^{2}$} \\
\hline Melanophores & 1 and 5 & + & - & + \\
\hline Xanthophores & 5 & + & + & + \\
\hline \multicolumn{5}{|l|}{ GOLDFISH $^{3}$} \\
\hline Xanthophores & 1 & + & ++ & ++ \\
\hline
\end{tabular}

Des, Des-Ac- $\alpha-M S H ;$ Mono, $\alpha-M S H ;$ Di, Di-Ac- $\alpha-M S H ; N$, not examined.

${ }^{1}$ Taken from Kobayashi et al. (2010).

${ }^{2}$ Taken from Kobayashi et al. (2012).

${ }^{3}$ Taken from Kobayashi et al. (2011). 
in humans (Schiöth et al., 1997). Therefore, we assumed that a receptor(s) similar to human MC4R and/or MC5R is generated in the melanophores of bf flounder skin. To verify this assumption, we examined mor expression using isolated skin cells. The results indicated that we needed to change our assumption because modulation of the pigment-dispersing activity was suggested to be associated with two different $\mathrm{mcr}$ subtypes expressed concomitantly, as described below.

Two chromatophore types, melanophores and xanthophores, are observed in bf flounder skin. These chromatophores and nonchromatophoric dermal cells were isolated from skin pieces and used to examine $m c r$ expression. When RT-PCR was performed for $m c 1 r, m c 2 r, m c 4 r$, and $m c 5 r$ using total RNA extracted from single cells of bf flounder fin skin, a cDNA fragment of $m c 5 r$, but not those of other subtypes, was amplified from the total RNA prepared from xanthophores (Table 1). As described above, $\alpha$-MSH exhibited higher pigment-dispersing activity than DesAc- $\alpha-\mathrm{MSH}$ did, suggesting that acetylation at the $\mathrm{N}$-terminus of $\alpha$-MSH increased the activity. Pharmacological studies on sea bass MC5R have revealed that $\alpha$-MSH efficacy in stimulating cellular activities is higher than that of Des-Ac- $\alpha-\mathrm{MSH}$ (Sánchez et al., 2009a). Acetylation-mediated augmentation of the binding affinity of Des-Ac- $\alpha$-MSH was also observed during pharmacological studies with human MC5R (Schiöth et al., 1997). Therefore, it is possible that acetylation of the ligand results in increased binding affinity of the ligand to MC5R, thus leading to increased pigment-dispersing activity.

In contrast, $\alpha-\mathrm{MSH}$ did not stimulate pigment dispersion in melanophores (Kobayashi et al., 2009). When RT-PCR was performed using total RNA extracted from single cells of bf flounder fin skin, cDNA fragments of $m c 1 r$ and $m c 5 r$, but not those of other subtypes, was amplified from the total RNA prepared from melanophores (Table 1). An apparent contradiction concerning pigment migration in xanthophores could be elucidated by the expression of two different $m c r$ subtypes in bf flounder melanophores. This idea came from increasing biochemical and biophysical evidences indicating that many GPCRs form dimers or higher orders of oligomers (Angers et al., 2002; Breitwieser, 2004; Kroeger et al., 2004; Nakata et al., 2005; Eglen et al., 2007; Milligan, 2007). Such receptor dimerization may occur not only between identical molecules that comprise homodimers, but also between related molecules belonging to the GPCR families. Heterodimerization may either increase or decrease the affinity of ligands to the corresponding GPCRs. For example, a heterodimer consisting of somatostatin receptor (SSTR) five and dopamine D2 receptor exhibits a high affinity for both SSTR and D2 agonists (Rocheville et al., 2000). On the other hand, a heterodimer consisting of SSTR 2A and SSTR3 inactivates SSTR3 function (Pfeiffer et al., 2001). According to these findings, it is plausible that MC1R and MC5R on bf flounder melanophores may constitute heterodimers. It is also conceivable that the affinity of $\alpha-\mathrm{MSH}$ with an acetyl group may be dramatically reduced by heterodimerization of the MCRs, whereas the affinity of Des-Ac- $\alpha-\mathrm{MSH}$ is retained (Figure 1).

\section{EVIDENCE OBTAINED FROM Jpn FLOUNDER}

We evaluated the results obtained with bf flounder using Jpn flounder, which is another member of Pleuronectiformes, to determine
A
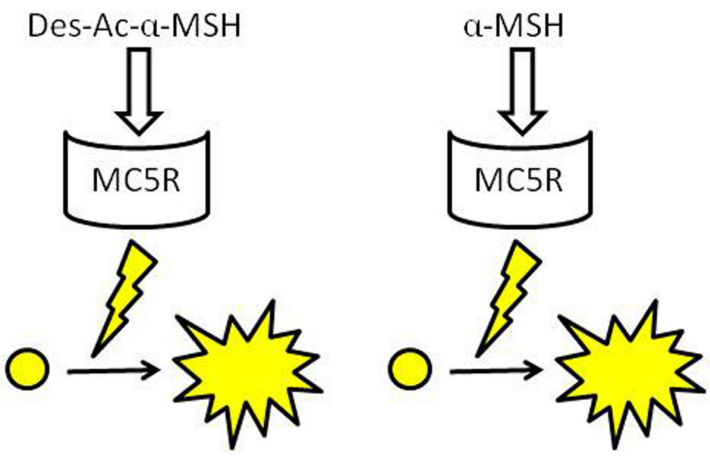

B

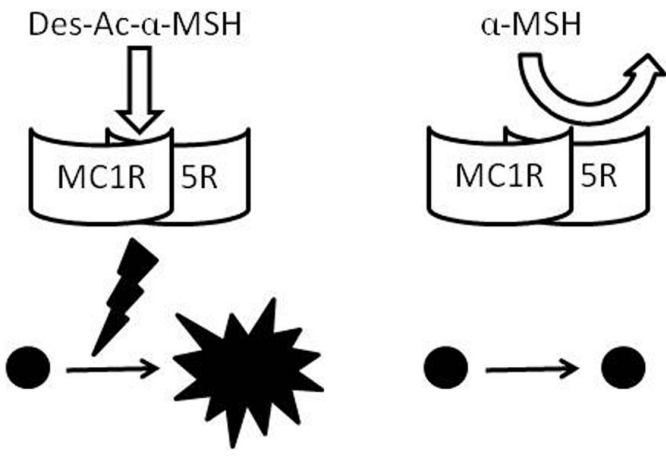

FIGURE 1 | Schematic models of the pigment-dispersing activities of $\alpha$-MSH-related peptides via MCRs. Both Des-Ac- $\alpha-M S H$ and $\alpha-M S H$ stimulate pigment dispersion when one type of MCR is expressed in chromatophores, such as in xanthophores of bf flounder, Jpn flounder, and goldfish (A). Des-Ac- $\alpha-M S H$ also exhibits activities in chromatophores expressing two types of MCRs, such as in the melanophores of bf and Jpn flounders, but $\alpha-\mathrm{MSH}$ does not. $\alpha-\mathrm{MSH}$ may have low affinity to putative MCR heterodimers (B).

whether relationships between the effects of $\alpha$-MSH-related peptides and mors expressed in chromatophores are restricted to bf flounder or are common to other species. In other words, we investigated whether reduction of the pigment-dispersing activities of $\alpha-\mathrm{MSH}$ is related to the expression of two different $m c r$ types.

The expression of only $m c 5 r$ among four $m c r$ subtypes was observed in Jpn flounder xanthophores. This result is identical to that obtained in bf flounder (Kobayashi et al., 2009). Differences between the two flounders were observed in the pigment dispersion in xanthophores; namely, $\alpha$-MSH activities were indistinguishable from those of Des-Ac- $\alpha-\mathrm{MSH}$ in Jpn flounder, while $\alpha$-MSH was more potent than Des-Ac- $\alpha$-MSH in bf flounder (Kobayashi et al., 2009). Thus, the pigment-dispersing activity of $\alpha-\mathrm{MSH}$ is not always enhanced by the presence of an acetyl group in chromatophores expressing a single $\mathrm{mcr}$ subtype, while pharmacological studies using sea bass MC5R expressed in human embryonic kidney (HEK) 293 cells have demonstrated that the response to $\alpha-\mathrm{MSH}$ is undoubtedly higher than the response to Des-Ac- $\alpha$-MSH (Sánchez et al., 2009a). These results suggest that signal transduction processes in relation to pigment migration between the xanthophores of bf and Jpn flounders are different, while $M c 5 r$ is expressed in these cells. 
Mc1r and mc5r transcripts were detected in Jpn flounder melanophores. This result is the same as that obtained from bf flounder (Kobayashi et al., 2010). The effects of $\alpha$-MSH-related peptides on pigment dispersion in Jpn flounder were also comparable to those observed in bf flounder; namely, Des-Ac- $\alpha-\mathrm{MSH}$ exhibited the activities, while $\alpha$-MSH never stimulated pigment dispersion (Kobayashi et al., 2009). Consequently, experiments using Jpn and bf flounders demonstrated that the remarkable reduction of pigment-dispersing activity is observed specifically in melanophores, in which two different mor types are concomitantly expressed. Therefore, it is possible that the physicochemical property of $\alpha$-MSH originating from the presence of an acetyl group causes the reduction of its activity, and this reduction appears to be associated with melanophore properties that differ from that of xanthophores with regard to ligand-receptor interaction. Results obtained from Jpn flounder support the occurrence of MCR heterodimers modifying the activity of $\alpha$-MSH-related peptides (Figure 1).

\section{EVIDENCE OBTAINED FROM GOLDFISH}

The pigment-dispersing activity of $\alpha-\mathrm{MSH}$ in goldfish xanthophores is somewhat greater than that of Des-Ac- $\alpha$-MSH. These results are similar to those observed in bf flounder xanthophores (Kobayashi et al., 2009). Only one $m c r$ subtype, i.e., $m c 1 r$ and $m c 5 r$ in goldfish and flounders, respectively, is expressed in the xanthophores. Considered together, $\alpha$-MSH-related peptides exhibit pigment-dispersing activities irrespective of the degree of acetylation when one type of $\mathrm{Mcr}$ is expressed (Table 1). Monoacetylation

\section{REFERENCES}

Amemiya, Y., Takahashi, A., Suzuki, N., Sasayama, Y., and Kawauchi, H. (1999). A Newly characterized melanotropin in proopiomelanocortin in pituitaries of an elasmobranch, Squalus acanthias. Gen. Comp. Endocrinol. 114, 387-395.

Amemiya, Y., Takahashi, A., Suzuki, N., Sasayama, Y., and Kawauchi, H. (2000). Molecular cloning of proopiomelanocortin cDNA from an elasmobranch, the stingray, Dasyatis akajei. Gen. Comp. Endocrinol. 118, 105-112.

Angers, S., Salahpour, A., and Bouvier, M. (2002). Dimerization: an emerging concept for $\mathrm{G}$ protein-coupled receptor ontogeny and function. Annu. Rev. Pharmacol. Toxicol. 42, 409-439.

Breitwieser, G. E. (2004). G proteincoupled receptor oligomerization: implications for $\mathrm{G}$ protein activation and cell signaling. Circ. Res. 94, $17-27$.

Castro, M. G., and Morrison, E. (1997). Post-translational processing of proopiomelanocortin in the pituitary and in the brain. Crit. Rev. Neurobiol. 11, 35-57.

Cerdá-Reverter, J. M., Ringholm, A., Schiöth, H. B., and Peter, R. E. (2003a). Molecular cloning, pharmacological characterization, and

of the N-terminus may contribute to an increase in the pigmentdispersing activity of $\alpha$-MSH-related peptides in goldfish because the $\alpha$-MSH activity was found to be slightly but significantly higher than that of Des-Ac- $\alpha-\mathrm{MSH}$ when their effects were compared at low concentrations. Similar enhancing effects of pigment dispersion caused by monoacetylation have also been observed in grass carp, tilapia, and frogs (Kawauchi et al., 1984; Ebelre, 1988; van der Salm et al., 2005). Only one mor subtype appears to be expressed in the melanophores of these species. Additionally, the pigment-dispersing activity of $\alpha-\mathrm{MSH}$ was comparable to that of Di-Ac- $\alpha-\mathrm{MSH}$. Pharmacological studies using sea bass MC1R expressed in HEK 293 cells have exhibited similar responses to both $\alpha$ - and Di-Ac- $\alpha$-MSH (Sánchez et al., 2010). Similar pharmacological properties have been observed for sea bass MC5R (Sánchez et al., 2009a). Therefore, it is suggested that both MC1R and MC5R recognize $\alpha-\mathrm{MSH}$ and $\mathrm{Di}-\mathrm{Ac}-\alpha-\mathrm{MSH}$ equally.

\section{CONCLUSION}

Based on comparative studies using chromatophores from flounders and goldfish, it is hypothesized that the pigment-dispersing activity of $\alpha-\mathrm{MSH}$ is reduced in chromatophores expressing two different $m c r$ subtypes due to the formation of MCR heterodimers. On the other hand, when one mor type is expressed, as in the case of flounder and goldfish xanthophores, $\alpha$-MSH-related peptides such as Des-Ac- $\alpha-\mathrm{MSH}, \alpha-\mathrm{MSH}$, and Di-Ac- $\alpha-\mathrm{MSH}$ exhibit pigmentdispersing activities in a dose-dependent manner irrespective of the degree of acetylation. Further experiments using a variety of fishes are necessary to confirm the hypothesis.

Haitina, T., Klovins, J., Takahashi, A., Löwgren, M., Ringholm, A., Enberg, J., Kawauchi, H., Larson, E. T., Fredriksson, R., and Schiöth, H. B. (2007a). Functional characterization of two melanocortin (MC) receptors in lamprey showing orthology to the $\mathrm{MC1}$ and $\mathrm{MC4}$ receptor subtypes. BMC Evol. Biol. 7, 101-114. doi:10.1186/1471-2148-7-101

Haitina, T., Takahashi, A., Holmén, L., Enberg, J., and Schiöth, H. B. (2007b). Further evidence for ancient role of $\mathrm{ACTH}$ peptides at melanocortin (MC) receptors; pharmacology of dogfish and lamprey peptides at dogfish MC receptors. Peptides 28, 798-805.

Kasper, R. S., Shved, N., Takahashi, A., Reinecke, M., and Eppler, E. (2006). A systematic immunohistochemical survey of the distribution patterns of $\mathrm{GH}$, prolactin, somatolactin, $\beta-\mathrm{TSH}$, $\beta$-FSH, $\beta$-LH, ACTH, and $\alpha-\mathrm{MSH}$ in the adenohypophysis of Oreochromis niloticus, the Nile tilapia. Cell Tissue Res. 325, 303-313.

Kawauchi, H., Kawazoe, I., Adachi, Y., Buckley, D. I., and Ramachandran, J. (1984). Chemical and biological characterization of salmon melanocyte-stimulating hormones. Gen. Comp. Endocrinol. 53, 37-48.
Kim, K. S., Kim, H.-W., Chen, T. T., and Kim, Y. T. (2009). Molecular cloning, tissue distribution and quantitative analysis of two proopiomelanocortin mRNAs in Japanese flounder (Paralichthys olivaceus). BMB Rep. 42, 206-211.

Klovins, J., Haitina, T., Fridmanis, D., Kilianova, Z., Kapa, I., Fredriksson, R., Gallo-Payet, N., and Schiöth, H. B. (2004). The Melanocortin system in fugu: determination of POMC/AGRP/MCR gene repertoire and synteny, as well as pharmacology and anatomical distribution of the MCRs. Mol. Biol. Evol. 21, 563-579.

Kobayashi, Y., Chiba, H., Amiya, N., Yamanome, T., Mizusawa, K., Amano, M., and Takahashi, A. (2008). Expression of proopiomelanocortin genes in pituitary gland of barfin flounder does not always respond to background color. Gen. Comp. Endocrinol. 158, 259-267.

Kobayashi, Y., Chiba, H., Mizusawa, K., Suzuki, N., José Miguel CerdáReverte, J. M., and Takahashi, A (2011). Pigment-dispersing activities and cortisol-releasing activities of melanocortins and their receptors in xanthophores and head kidneys of the goldfish Carassius auratus. Gen. Comp. Endocrinol. 173, 438-446. 
Kobayashi, Y., Mizusawa, K., Chiba, H., Tagawa, M., and Takahashi, A. (2012). Further evidence on the cancellation of $\alpha$-melanocytestimulating hormone activity by acetylation in melanophores using Japanese flounder. Gen. Comp. Endocrinol. (in press).

Kobayashi,Y., Mizusawa, K., Yamanome, T., Chiba, H., and Takahashi, A. (2009). Possible paracrine function of $\alpha$-melanocyte-stimulating hormone and inhibition of its melanin-dispersing activity by $\mathrm{N}$ terminal acetylation in the skin of the barfin flounder, Verasper moseri. Gen. Comp. Endocrinol. 161, 419-424.

Kobayashi, Y., Okada, S., Tsuchiya, K., Yamanome, T., and Takahashi, A. (2007). "Distribution of melanocortin receptors and effects of feeding on its expression within barfin flounder, Pleuronectiformes," in Proceedings of the Japan Society for Comparative Endocrinology No. 22, eds S. Tanaka and M. Suzuki (Tokyo: Japan Society of Comparative Endocrinology), 29.

Kobayashi, Y., Tsuchiya, K., Yamanome, T., Schiöth, H. B., and Takahashi, A. (2010). Differential expressions of melanocortin receptor subtypes in melanophores and xanthophores of barfin flounder. Gen. Comp. Endocrinol. 168, 133-142.

Kroeger, K. M., Pfleger, K. D. G., and Eidne, K. A. (2004). G-protein coupled receptor oligomerrization in neuroendocrine pathways. Front. Neuroendocrinol. 24, 254-278.

Lamers, A. E., Flik, G., Atsma, W., and Wendelaar Bonga, S. E. (1992). A role for di-acetyl- $\alpha$-melanocytestimulating hormone in the control of cortisol release in the teleost Oreochromis mossambicus. J. Endocrinol. 135, 285-292.

Logan, D. W., Bryson-Richardson, R. J., Pagan, K. E., Taylor, M. S., Currie, P. D., and Jackson, I. J. (2003). The structure and evolution of the melanocortin and $\mathrm{MCH}$ receptors in fish and mammals. Genomics 81, 184-191.

Milligan, G. (2007). G protein-coupled receptor dimerisation: molecular basis and relevance to function. Biochim. Biophys. Acta 1768, 825-835.

Mizusawa, K., Kobayashi, Y., Sunuma, T., Asahida, T., Saito, Y., and Takahashi, A. (2011). Inhibiting roles of melanin-concentrating hormone for skin pigment dispersion in barfin flounder, Verasper moseri. Gen. Comp. Endocrinol. 171, 75-81.

Mountjoy, K. G., Robbins, L. S., Mortud, M. T., and Cone, R. D. (1992). The cloning of a family of genes that encode the melanocortin receptors. Science 257. 1248-1251.

Mountjoy, K. G. (2000). "Cloning of the melanocortin receptors," in The Melanocortin Receptors, ed. R. D. Cone (Totowa, NJ: Human Press), 209-235.

Nakata, H., Yoshioka, K., Kamiya, T., Tsuga, H., and Oyanagi, K. (2005). Functions of heteromeric association between adenosine and $\mathrm{P} 2 \mathrm{Y}$ receptors. J. Mol. Neurosci. 26, 233-238.

Nelson, J. S. (2006). Fishes of the World, 4th Edn. New York: John Wiley and Sons.

Pfeiffer, M., Koch, T., Schröder, H., Klutzny, M., Kirscht, S., Kreienkamp, H. J., Höllt, V., and Schulz, S. (2001). Homo- and heterodimerization of somatostatin receptor subtypes. Inactivation of sst(3) receptor function by heterodimerization with sst(2A). J. Biol. Chem. 276, 14027-14036.

Raffin-Sanson, M. L., de Keyzer, Y., and Brtagna, X. (2003). Proopiomelanocortin, a polypeptide precursor with multiple functions: from physiology to pathological conditions. Eur. J. Endocrinol. 149, 79-90.

Rocheville, M., Lange, D. C., Kumar, U., Patel, S. C., Patel, R. C., and Patel, Y. C. (2000). Receptors for dopamine and somatostatin: formation of hetero-oligomers with enhanced functional activity. Science 288, 154-157.

Rozenfeld, R., and Devi, L. A. (2011). Exploring a role for heteromerization in GPCR signaling specificity. Biochem. J. 433, 11-18.

Saito, Y., Nothacker, H. P., Wang, Z., Lin, S. H., Leslie, F., and Civelli, O. (1999). Molecular characterization of the melanin-concentratinghormone receptor. Nature 400, 265-269.

Sánchez, E., Rubio, V. C., and CerdáReverter, J. M. (2009a). Characterization of the sea bass melanocotin 5 receptor: a putative role in hepatic lipid metabolism. J. Exp. Biol. 212, 3901-3910.

Sánchez, E., Rubio, V. C., Thompson, D., Met, J., Flik, G., Millhauser, G. L., and Cerdá-Reverter, J. M. (2009b). Phosphodiesterase inhibitor-dependent inverse agonism of agouti-related protein on melanocortin 4 receptor in sea bass
(Dicentrarchus labrax). Am. J. Physiol. Regul. Integr. Comp. Physiol. 296 R1293-R1306.

Sánchez, E., Rubio, V. C., and CerdáReverter, J. M. (2010). Molecular and pharmacological characterization of the melanocortin type 1 receptor in sea bass. Gen. Comp. Endocrinol. 165 163-169.

Satake, H., and Sakai, T. (2008). Recent advances and perceptions in studies of heterodimerization between $\mathrm{G}$ protein-coupled receptors. Protein Pept. Lett. 15, 300-308.

Schiöth, H. B., Haitina, T., Ling, M. K., Ringholm, A., Fredriksson, R. Cerdá-Reverter, J. M., and Klovins, J. (2005). Evolutionary conservation of the structural, pharmacological, and genomic characteristics of the melanocortin receptor subtypes. Peptides 26, 1886-1990.

Schiöth, H. B., Muceniece, R., Larsson, M., and Wikberg, J. E. S. (1997). The melanocortin 1, 3,4 or 5 receptors do not have a binding epitope for ACTH beyond the sequence of $\alpha-\mathrm{MSH}$. J. Endocrinol. 155, 73-78.

Schiöth, H. B., Muceniece, R., and Wikberg, J. E. S. (1996). Characterisation of the melanocortin 4 receptor by radioligand binding. Pharmacol. Toxicol. 79, 161-165.

Smith, A. I., and Funder, J. W. (1988) Proopiomelanocortin processing in the pituitary, central nervous system, and peripheral tissues. Endocr. Rev. 9, 159-179.

Sugimoto, M. (2002). Morphological color changes in fish: regulation of pigment cell density and morphology. Microsc. Res. Tech. 58, 496-503.

Takahashi, A., Amano, M., Amiya, N. Yamanome, T., Yamamori, K., and Kawauchi, H. (2006). Expression of three proopiomelanocortin subtype genes and mass spectrometric identification of POMC-derived peptides in pars distalis and pars intermedia of barfin flounder pituitary. Gen. Comp. Endocrinol. 145 280-286.

Takahashi, A., Amano, M., Itoh, T. Yasuda, A., Yamanome, T., Amemiya, Y., Sasaki, K., Sakai, M., Yamamori, K., and Kawauchi, H. (2005). Nucleotide sequence and expression of three subtypes of proopiomelanocortin mRNA in barfin flounder. Gen. Comp. Endocrinol. 141. 291-303.

Takahashi, A., Itoh, T., Nakanishi, A., Amemiya, Y., Ida, H., Meguro, H., and Kawauchi, H. (2004). Molecular cloning of proopiomelanocortin
cDNA in the ratfish, a holocephalan. Gen. Comp. Endocrinol. 135, 159-165.

Takahashi, A., and Kawauchi, $\mathrm{H}$. (2006a). Evolution of melanocortin systems in fish. Gen. Comp. Endocrinol. 148, 85-94.

Takahashi, A., and Kawauchi, $H$. (2006b). "Diverse structures and functions of melanocortin, endorphin and melanin-concentrating hormone in fish," in Fish Endocrinol ogy, eds G. Zaccone, M. Reinecke, and B. G. Kapoor (Enfield, NH: Science Publishers), 325-392.

Takahashi, A., Kobayashi, Y., Moriyama, S., and Hyodo, S. (2008). Evaluation of posttranslational processing of proopiomelanocortin in the banded houndshark pituitary by combined cDNA cloning and mass spectrometry. Gen. Comp. Endocrinol. 157, 41-48.

van der Salm, A. L., Metz, J. R., Wendellar Bonga, S. E., and Flik, G. (2005) Alpha-MSH, the melanocortin-1 receptor and background adaptation in the Mozambique tilapia, Oreochromis mossambicus. Gen. Comp. Endocrinol. 144, 140-149.

Yada, T., Azuma, T., Takahashi, A., Suzuki, Y., and Hirose, S. (2000). Effects of desacetyl $\alpha$-MSH on lipid mobilization in the rainbow trout, Oncorhynchus mykiss. Zool. Sci. 17, 1123-1127.

Conflict of Interest Statement: The authors declare that the research was conducted in the absence of any commercial or financial relationships that could be construed as a potential conflict of interest.

Received: 07 September 2011; accepted: 11 January 2012; published online: 01 February 2012

Citation: Kobayashi Y, Mizusawa K, Saito $Y$ and Takahashi A (2012) Melanocortin systems on pigment dispersion in fish chromatophores. Front. Endocrin. 3:9. doi: 10.3389/fendo.2012.00009

This article was submitted to Frontiers in Experimental Endocrinology, a specialty of Frontiers in Endocrinology.

Copyright () 2012 Kobayashi, Mizusawa, Saito and Takahashi. This is an openaccess article distributed under the terms of the Creative Commons Attribution Non Commercial License, which permits non-commercial use, distribution, and reproduction in other forums, provided the original authors and source are credited. 